JOURNAL OF INTEGRAL EQUATIONS AND APPLICATIONS

Volume 8, Number 3, Summer 1996

\title{
IMPROVED CONVERGENCE RATES FOR SOME DISCRETE GALERKIN METHODS
}

\author{
MICHAEL GOLBERG
}

\begin{abstract}
We show how to improve the estimate of the convergence rate of a number of discrete polynomiallybased Galerkin methods for Fredholm and Cauchy singular integral equations. This has been accomplished by sharpening the bounds on the quadrature errors in a manner analogous to that of Joe [14] for spline-based methods. These results are then extended to establish the convergence of some discrete Galerkin methods for one-dimensional hypersingular equations and some boundary integral equations on the sphere in $\mathbf{R}^{3}$.
\end{abstract}

Introduction. In a number of recent papers we have examined the convergence rate of various polynomially-based Galerkin methods for Fredholm and singular integral equations [8-12]. The convergence analysis took into account the effects of quadrature errors and for Fredholm equations may be seen as complementary to similar results of Atkinson and Bogomolny [4], Joe [14] and Spence and Thomas [20] using spline bases. In the case of splines, the above authors were able to obtain optimal convergence rates, i.e., convergence rates equal to that of the best approximation to the solution by splines of a given order. For polynomial approximations we were unable to do this, in part because of over estimation of various quadrature errors. In this paper, making use of an argument analogous to that of Joe [14] for spline approximations, we are able to improve our estimate of the convergence rate from $0\left(n^{-r+1}\right)$ to $0\left(n^{-r+\frac{1}{2}}\right)$ where $n$ is the degree of the polynomial approximation. This seems the best that can be done by perturbation techniques.

The paper is divided into five sections. In Section 2 we review our previous results for Fredholm and Cauchy singular equations and indicate where improvements to our prior analysis can be made. In Section 3 we provide new estimates of quadrature errors generalizing those in $[\mathbf{1 0}-\mathbf{1 2}]$. These are then applied to improve the convergence rates

Received by the editors on November 25, 1994, and in revised form on January 28,1996 .

Copyright (C)1996 Rocky Mountain Mathematics Consortium 
given in $[\mathbf{1 0 - 1 2}]$. In Section 4 we extend these results to establish the convergence of a discrete Galerkin method for hypersingular integral equations discussed by Frenkel $[6]$ and us in $[\mathbf{1 0}, \mathbf{1 1}]$. In Section 5 we analyze the convergence of some discrete Galerkin methods based on spherical harmonics for integral equations on the sphere.

\section{Discrete Galerkin methods.}

2.1 Fredholm equations. We consider the numerical solution of the integral equation

$$
u(x)=\int_{-1}^{1} \kappa(x, t) u(t) d t+f(x)
$$

where $\kappa(x, t)$ is continuous on $[-1,1] \times[-1,1]$ and $f(x)$ is continuous on $[-1,1]$. We assume that $(2.1)$ has a unique solution. Let $L_{2}$ be the space of real square-integrable functions on $[-1,1]$ with inner product

$$
\langle f, g\rangle=\int_{-1}^{1} f(t) g(t) d t
$$

and norm

$$
\|f\|=(\langle f, f\rangle)^{1 / 2} .
$$

To solve (2.1) by Galerkin's method let $\left\{\varphi_{k}\right\}$ be the normalized Legendre polynomials and approximate $u$ by

$$
u_{n}=\sum_{k=0}^{n} a_{k} \varphi_{k}
$$

where the coefficients $\left\{a_{k}\right\}_{k=0}^{n}$ are determined by solving

$$
a_{j}-\sum_{k=0}^{n}\left\langle K \varphi_{k}, \varphi_{j}\right\rangle a_{k}=\left\langle f, \varphi_{j}\right\rangle, \quad j=0,1, \ldots, n
$$

and

$$
K u(x)=\int_{-1}^{1} \kappa(x, t) u(t) d t
$$


Under the stated conditions on $\kappa$ and $f$ it is well known that for all $n$ sufficiently large that $u_{n}$ exists, is unique and

$$
\left\|u-u_{n}\right\| \leq c n^{-r}
$$

if $\kappa \in C^{r}$ and $f \in C^{r}$, thus establishing the convergence of $u_{n}$ to $u$.

One can also show that $u_{n}$ converges uniformly to $u$ if $r \geq 2$ and [12]

$$
\left\|u-u_{n}\right\|_{\infty} \leq c n^{-r+3 / 2}
$$

where $\|\cdot\|_{\infty}$ is the uniform norm.

In general the integrals in (2.5) cannot be evaluated analytically, so some type of numerical integration method needs to be used to obtain approximations to $\left\langle f, \varphi_{j}\right\rangle$ and $\left\langle K \varphi_{k}, \varphi_{j}\right\rangle$. For this we define integration rules $\left\{Q_{M(n)}\right\}$

$$
\int_{-1}^{1} g(t) d t \simeq Q_{M(n)}(g)=\sum_{l=1}^{M} w_{l} g\left(t_{l}\right)
$$

where

(1) $w_{l}>0, l=1,2, \ldots, M(n)$;

(2) $Q_{M(n)}$ has precision at least $2 n$; that is,

$$
Q_{M(n)}(g)=\int_{-1}^{1} g(t) d t
$$

for all polynomials of degree $\leq 2 n$. (For simplicity we will suppress the dependence of $M(n)$ on $n$ for the remainder of the paper.)

Using $Q_{M}$ we define the following approximations:

$$
\left\langle f, \varphi_{k}\right\rangle \simeq Q_{M}\left(f \varphi_{k}\right), \quad 0 \leq k \leq n,
$$

and

$$
\begin{aligned}
\left\langle K \varphi_{k}, \varphi_{j}\right\rangle & =\int_{-1}^{1} \int_{-1}^{1} \kappa(x, t) \varphi_{k}(t) \varphi_{j}(x) d x d t \\
& \simeq \sum_{m=1}^{M} \sum_{l=1}^{M} w_{l} w_{m} \kappa\left(x_{m}, t_{l}\right) \varphi_{k}\left(t_{l}\right) \varphi_{j}\left(x_{m}\right) \\
& \equiv Q_{M} \times Q_{M}\left(\kappa \varphi_{k} \varphi_{j}\right)
\end{aligned}
$$


where $\left\{x_{m}\right\}$ and $\left\{t_{l}\right\}$ represent the same sets of points.

Substituting (2.11)-(2.12) into (2.5) and letting $v_{n}$ be the resulting approximation to $u_{n}$

$$
v_{n}=\sum_{k=0}^{n} b_{k} \varphi_{k}
$$

where $\left\{b_{k}\right\}_{k=0}^{n}$ satisfy

$$
\begin{aligned}
b_{j}-\sum_{k=0}^{n} b_{k}\left[\sum_{m=1}^{M} \sum_{l=1}^{M}\right. & \left.w_{m} w_{l} \kappa\left(x_{m}, t_{l}\right) \varphi_{k}\left(t_{l}\right) \varphi_{j}\left(x_{m}\right)\right] \\
& =\sum_{m=1}^{M} w_{m} f\left(x_{m}\right) \varphi_{j}\left(x_{m}\right), \quad 0 \leq j \leq n .
\end{aligned}
$$

The method defined by (2.13)-(2.14) is called a discrete Galerkin method.

To prove the convergence of $v_{n}$ and to obtain rates of convergence we use the theory of perturbed projection methods as in [12]. Then some tedious algebra shows that $v_{n}$ defined by (2.13)-(2.14) satisfies the operator equation

$$
v_{n}=\pi_{n} K_{n} v_{n}+\pi_{n} f,
$$

where

$$
K_{n} u(x)=\sum_{l=1}^{M} w_{l} \kappa\left(x, t_{l}\right) u\left(t_{l}\right)
$$

and $\pi_{n}$ is a 'discrete projection'

$$
\pi_{n} u(x)=\sum_{k=0}^{n} Q_{M}\left(u \varphi_{k}\right) \varphi_{k}(x) .
$$

Letting $P_{n}$ be the operator of orthogonal projection onto $X_{n}=$ span $\left(\left\{\varphi_{k}\right\}_{k=0}^{n}\right)$, some rearrangement of $(2.15)$ gives

$$
v_{n}=P_{n} K v_{n}+R_{n} v_{n}+P_{n} f+r_{n}
$$


where

$$
R_{n} v_{n}=-\left(P_{n} K v_{n}-\pi_{n} K_{n} v_{n}\right)
$$

and

$$
r_{n}=-\left(P_{n} f-\pi_{n} f\right) .
$$

Note that $R_{n}$ may be viewed as an operator from $X_{n} \rightarrow X_{n}$ and $v_{n} \in X_{n}$.

Using (2.17), (2.20) and the fact that

$$
\begin{gathered}
P_{n} f=\sum_{k=0}^{n}\left\langle f, \varphi_{k}\right\rangle \varphi_{k} \\
r_{n}=-\sum_{k=0}^{n}\left\langle f, \varphi_{k}\right\rangle \varphi_{k}+\sum_{k=0}^{n} Q_{M}\left(f \varphi_{k}\right) \varphi_{k} \\
=-\left[\sum_{k=0}^{n}\left(\left\langle f, \varphi_{k}\right\rangle-Q_{M}\left(f \varphi_{k}\right)\right) \varphi_{k}\right] \\
=-\sum_{k=0}^{n} e_{k}\left(f \varphi_{k}\right) \varphi_{k}
\end{gathered}
$$

where $e_{k}\left(f \varphi_{k}\right)=\left\langle f, \varphi_{k}\right\rangle-Q_{M}\left(f \varphi_{k}\right)$ is the integration error in approximating $\left\langle f, \varphi_{k}\right\rangle$ by $Q_{M}\left(f \varphi_{k}\right)$.

Similarly

$$
R_{n} v_{n}=\sum_{k=0}^{n} E_{k}\left(\kappa v_{n} \varphi_{k}\right) \varphi_{k}
$$

where $E_{k}\left(\kappa v_{n} \varphi_{k}\right)$ is the integration error in approximating $\left\langle K v_{n}, \varphi_{k}\right\rangle$ by $Q_{M} \times Q_{M}\left(\kappa v_{n} \varphi_{k}\right)$.

Letting

$$
\left\|R_{n}\right\|_{n}=\operatorname{lub}\left\{\left\|R_{n} w_{n}\right\|, w_{n} \in X_{n},\left\|w_{n}\right\|=1\right\}
$$


it follows from Theorem 1 of [18] that if $\left\|R_{n}\right\|_{n} \rightarrow 0$ and $\left\|r_{n}\right\| \rightarrow 0$ that, for all $n$ sufficiently large, $v_{n} \rightarrow u$ and

$$
\left\|u-v_{n}\right\| \leq c\left[\left\|u-u_{n}\right\|+\left\|R_{n}\right\|_{n}+\left\|r_{n}\right\|\right] .
$$

Since $\left\|u-u_{n}\right\|$ is bounded by (2.7), it suffices to bound $\left\|R_{n}\right\|_{n}$ and $\left\|r_{n}\right\|$ in order to bound $\left\|u-v_{n}\right\|$. In [12] we used the bounds

$$
\left\|r_{n}\right\|=\left[\sum_{k=0}^{n} e_{k}^{2}\left(f \varphi_{k}\right)\right]^{1 / 2}
$$

and

$$
\left\|R_{n}\right\|_{n} \leq\left[\sum_{k=0}^{n} \sum_{j=0}^{n} E_{j k}^{2}\right]^{1 / 2}
$$

where

$$
E_{j k}=\left\langle K \varphi_{k}, \varphi_{j}\right\rangle-Q_{M} \times Q_{M}\left(\kappa \varphi_{k} \varphi_{j}\right)
$$

is the integration error in approximating $\left\langle K \varphi_{k}, \varphi_{j}\right\rangle$ by $Q_{M} \times Q_{M}\left(\kappa \varphi_{k} \varphi_{j}\right)$.

In [12] (2.27) was obtained by expanding $w_{n}=\sum_{k=0}^{n} c_{k} \varphi_{k}, \sum_{k=0}^{n} c_{k}^{2}=$ 1 so that

$$
R_{n} w_{n}=\sum_{k=0}^{n} \sum_{j=0}^{n} E_{j k}\left(\kappa \varphi_{k} \varphi_{j}\right) \varphi_{k}
$$

and then using the Cauchy-Schwarz inequality. From this and the results for the integration errors given in [12] we found that $\left\|r_{n}\right\| \leq$ $c n^{-r+1 / 2}$ if $f \in C^{r}$, and $\left\|R_{n}\right\|_{n} \leq c n^{-r+1}$ if $\kappa \in C^{r}, r>1$. This gave

$$
\left\|u-v_{n}\right\| \leq c n^{-r+1}, \quad r>1 .
$$

However, if we do not expand $w_{n}$ as above, then

$$
\left\|R_{n}\right\|_{n}=l u b_{\left\{\left\|w_{n}\right\|=1\right\}}\left[\sum_{k=0}^{n} E_{k}^{2}\left(\kappa w_{n} \varphi_{k}\right)\right]^{1 / 2} .
$$

By modifying the proof in $[\mathbf{1 2}]$ it will be shown in Section 3 that

$$
\left|E_{k}\right| \leq n^{-r}
$$


for all $w_{n} \in X_{n}$ such that $\left\|w_{n}\right\|=1$. It then follows from (2.30) that $\left\|R_{n}\right\|_{n} \leq c n^{-r+1 / 2}$, the same bound as for $\left\|r_{n}\right\|$. Thus (2.25) gives

$$
\left\|u-v_{n}\right\| \leq c n^{-r+1 / 2}
$$

an improvement over the bound $\left\|u-v_{n}\right\| \leq c n^{-r+1}$ given in (2.29).

Unfortunately the bound in (2.32) still appears not to be optimal since it was shown in [12] that $\left\|u-v_{n}\right\| \leq c n^{-r}$ in the special case that $Q_{M}$ is $n+1$ point Gaussian quadrature. On the other hand, it seems that $(2.32)$ is the best that can be obtained by perturbation methods.

2.2 Cauchy singular equations. Here we consider solving the Cauchy singular integral equation (CSIE)

$$
a v(x)+\frac{b}{\pi} f_{-1}^{1} \frac{v(t)}{t-x} d t+\int_{-1}^{1} \kappa(x, t) v(t) d t=f(x),
$$

where the first integral in (2.33) is a Cauchy principal value, and for convenience we assume $a^{2}+b^{2}=1$. When $\kappa(x, t)=0$, it is well known that the solution of (2.33) is given by

$$
v(x)=w(x) u(x),
$$

where

$$
\begin{aligned}
w(x) & =(1-x)^{\alpha}(1+x)^{\beta}, \\
\alpha & =\frac{1}{2(\pi i)} \log \left[\frac{a-i b}{a+i b}\right]+N, \\
\beta & =-\frac{1}{2(\pi i)} \log \left[\frac{a-i b}{a+i b}\right]+M,
\end{aligned}
$$

and $M$ and $N$ are integers determined so that the index $\nu=-(\alpha+\beta)=$ $-(N+M)$ is restricted to the values $-1,0,1$. This guarantees that the solution to (2.33) is integrable if $f(x)$ is Hölder continuous. For simplicity we only consider the case $\nu=0$. (For the case $\nu=1$ we refer the reader to $[\mathbf{1 0}, \mathbf{1 1}]$. The analysis for $\nu=-1$ seems not to be in the literature.) 
The representation $v(x)=w(x) u(x)$ when $\kappa=0$ suggests the substitution $v(x)=w(x) u(x)$ in (2.33) with $u(x)$ satisfying

$$
\begin{aligned}
a w(x) u(x)+\frac{b}{\pi} \int_{-1}^{1} \frac{w(t) u(t)}{t-x} & d t \\
& +\int_{-1}^{1} w(t) \kappa(x, t) u(t) d t=f(x) .
\end{aligned}
$$

For convenience we write (2.38) in operator form

$$
H u+K u=f,
$$

where

$$
H u(x)=a w(x) u(x)+\frac{b}{\pi} f_{-1}^{1} \frac{w(t) u(t) d t}{t-x}
$$

and

$$
K u(x)=\int_{-1}^{1} w(t) \kappa(x, t) u(t) d t
$$

We assume that (2.39) has a unique solution.

To solve (2.39) by Galerkin's method, let $\left\{\psi_{k}\right\}_{k=0}^{\infty}$ be a set of Jacobi polynomials for $w(x)$ normalized so that

$$
\int_{-1}^{1} w(x) \psi_{k}^{2}(x) d x=1
$$

Similarly let $\left\{\chi_{k}\right\}_{k=0}^{\infty}$ be a set of Jacobi polynomials for $1 / w(x)$ normalized so that

$$
\int_{-1}^{1}\left[\chi_{k}^{2}(x) / w(x)\right] d x=1
$$

Then it is known that $\left\{\chi_{k}\right\}$ can be chosen so that [11]

$$
H \psi_{k}=\chi_{k}, \quad k \geq 0 .
$$


Now let $L_{w}$ denote the space of real functions square-integrable with respect to $w$, and similarly $L_{1 / w}$ is the space of functions square integrable with respect to $1 / w$. The inner product on $L_{\rho}, \rho=w$, or $1 / w$ is given by

$$
\langle f, g\rangle_{\rho}=\int_{-1}^{1} \rho(t) f(t) g(t) d t
$$

and the corresponding norm by $\|\cdot\|_{\rho}$.

From (2.44) it follows that $H$ can be extended as a bounded invertible operator from $L_{w} \rightarrow L_{1 / w}$. If $\kappa(x, t)$ is continuous, then $k$ defines a compact operator $K: L_{w} \rightarrow L_{1 / w}$. We assume this to be the case for the remainder of the paper. Thus $H+K$ may be considered as a bounded linear operator from $L_{w} \rightarrow L_{1 / w}$. Hence, we consider (2.39) as the problem of finding a unique $u \in L_{w}$ for each $f \in L_{1 / w}$.

Using these preliminaries we look for approximations to $u$ given by

$$
u_{n}=\sum_{k=0}^{n} a_{k} \psi_{k}
$$

with $\left\{a_{k}\right\}_{k=0}^{n}$ determined by setting the residual $\delta_{n}=(H+K) u_{n}-f$ orthogonal to $\left\{\chi_{j}\right\}_{j=0}^{n}$ in the $L_{1 / w}$ inner product, i.e.,

$$
\left\langle H u_{n}+K u_{n}-f, \chi_{j}\right\rangle_{1 / w}=0, \quad 0 \leq j \leq n .
$$

Using (2.44) and the fact that $\left\langle\chi_{k}, \chi_{j}\right\rangle_{1 / w}=\delta_{k j},(2.47)$ becomes

$$
a_{j}+\sum_{k=0}^{n}\left\langle K \psi_{k}, \chi_{j}\right\rangle_{1 / w} a_{k}=\left\langle f, \chi_{j}\right\rangle_{1 / w}, \quad 0 \leq j \leq n .
$$

In [11] it was shown that if $\kappa$ is continuous, then for all $n$ sufficiently large $u_{n}$ exists, is unique and

$$
\left\|u-u_{n}\right\|_{w} \leq c\left\|H u-\mathcal{Q}_{n} H u\right\|_{1 / w}
$$

where $\mathcal{Q}_{n}$ is the orthogonal projection of $L_{1 / w}$ onto $Y_{n}=$ span $\left(\left\{\chi_{j}\right\}_{j=0}^{n}\right)$. Since $\mathcal{Q}_{n} g \rightarrow g$ for all $g \in L_{1 / w}$, it follows from (2.49) with $g=H u$ that $u_{n} \rightarrow u$ in $L_{w}$. 
To get convergence rates, assume that $f \in C^{r}$ and $k \in C^{r}$, then $H u=f-K u \in C^{r}$ and it follows from (2.49) and Jackson's theorem [5] that

$$
\left\|u-u_{n}\right\|_{w} \leq c n^{-r}
$$

which is analogous to (2.7) for Fredholm equations.

However, as for Fredholm equations, the practical implementation of (2.46) and (2.48) requires that the inner products in (2.48) be evaluated numerically. For this we introduce quadrature rules $Q_{N}$ and $Q_{M}$ where

$$
\int_{-1}^{1}[g(x) / w(x)] d x \cong Q_{N}(g)=\sum_{m=1}^{N} \sigma_{m} g\left(x_{m}\right)
$$

and

$$
\int_{-1}^{1} w(t) g(t) d t \cong Q_{M}(g)=\sum_{l=1}^{M} w_{l} g\left(t_{l}\right)
$$

In addition, we require that

(1) $\quad \sigma_{m}>0, \quad 1 \leq m \leq N, \quad w_{l}>0, \quad 1 \leq l \leq M ;$

(2) $Q_{N}(g)=\int_{-1}^{1}[g(x) / w(x)] d x, \quad \forall g \in Y_{2 n}$,

$$
\text { (3) } \quad Q_{M}(g)=\int_{-1}^{1} w(x) g(x) d t, \quad \forall g \in X_{2 n} \text {. }
$$

Then we approximate

$$
\left\langle f, \chi_{j}\right\rangle_{1 / w} \simeq Q_{N}\left(f \chi_{j}\right)
$$

and

$$
\left\langle K \psi_{k}, \chi_{j}\right\rangle_{1 / w} \simeq Q_{M} \times Q_{N}\left(\kappa \psi_{k} \chi_{j}\right)
$$


In [10] we analyzed the convergence of the approximation $v_{n}$ to $u_{n}$ given by replacing the integrals in $(2.48)$ by $(2.55)-(2.56)$. For this we observe that

$$
v_{n}=\sum_{k=0}^{n} b_{k} \psi_{k}
$$

where $\left\{b_{k}\right\}_{k=0}^{n}$ satisfy

$$
\begin{aligned}
b_{j}+\sum_{k=0}^{n}\left[\sum_{m=1}^{N} \sum_{l=1}^{M} \sigma_{m} w_{l} \kappa\left(x_{m}, t_{l}\right) \psi_{k}\left(t_{l}\right)\right. & \left.\chi_{j}\left(x_{m}\right)\right] b_{k} \\
& =\sum_{m=1}^{N} \sigma_{m} f\left(x_{m}\right) \chi_{j}\left(x_{m}\right) .
\end{aligned}
$$

Letting

$$
\pi_{n} u=\sum_{k=0}^{n} Q_{N}\left(u \chi_{k}\right) \chi_{k}
$$

and

$$
K_{n} u=\sum_{l=1}^{M} w_{l} \kappa\left(x, t_{l}\right) u\left(t_{l}\right),
$$

it was shown in $[\mathbf{1 0}]$ that $(2.57)-(2.58)$ are equivalent to the operator equation

$$
H v_{n}+\mathcal{Q}_{n} K v_{n}+R_{n} v_{n}=\mathcal{Q}_{n} f+r_{n}
$$

where

$$
R_{n} v_{n}=-\left[\mathcal{Q}_{n} K v_{n}-\pi_{n} K_{n} v_{n}\right]
$$

and

$$
r_{n}=-\left[\mathcal{Q}_{n} f-\pi_{n} f\right],
$$

where $R_{n}: X_{n} \rightarrow Y_{n}$ and $r_{n} \in Y_{n}$. 
As for the Fredholm case, it can be shown that

$$
R_{n} v_{n}=-\sum_{k=0}^{n} E_{k}\left(\kappa \chi_{k} v_{n}\right) \chi_{k}
$$

and

$$
r_{n}=-\sum_{k=0}^{n} e_{k}\left(f \chi_{k}\right) \chi_{k}
$$

where $E_{k}\left(\kappa \chi_{k} v_{n}\right)$ is the integration error in approximating $\left\langle K v_{n}, \chi_{k}\right\rangle_{1 / w}$ by $Q_{M} \times Q_{N}\left(\kappa \chi_{k} v_{n}\right)$ and $e_{k}\left(f \chi_{k}\right)$ is the error in approximating $\left\langle f, \chi_{k}\right\rangle_{1 / w}$ by $Q_{N}\left(f \chi_{k}\right)$.

Using the result in [18] convergence of $v_{n} \rightarrow u$ is guaranteed if $\left\|R_{n}\right\|_{n} \rightarrow 0$ and $\left\|r_{n}\right\|_{1 / w} \rightarrow 0$ where

$$
\left\|R_{n}\right\|_{n}=l u b_{\left\{\left\|w_{n}\right\|_{w}=1\right\}}\left\{\left\|R_{n} w_{n}\right\|_{1 / w}\right\} .
$$

In $[\mathbf{1 0}]$ we used the bound

$$
\left\|R_{n}\right\|_{n} \leq\left[\sum_{k=0}^{n} \sum_{j=0}^{n} E_{j k}^{2}\right]^{1 / 2}
$$

where $E_{j k}=\left\langle K \psi_{j}, \chi_{k}\right\rangle_{1 / w}-Q_{M} \times Q_{N}\left(\kappa \psi_{j} \chi_{k}\right)$. For $k(x, t) \in C^{r}$ this gave the error bound $\left\|R_{n}\right\|_{n} \leq c n^{-r+1}$ [10]. Similarly, $\left\|r_{n}\right\|_{1 / w} \leq$ $c n^{-r+1 / 2}$ and using this in the estimate

$$
\left\|u-v_{n}\right\|_{w} \leq c\left[\left\|u-u_{n}\right\|_{w}+\left\|R_{n}\right\|_{n}+\left\|r_{n}\right\|_{1 / w}\right]
$$

gives

$$
\left\|u-v_{n}\right\|_{w} \leq c n^{-r+1}
$$

To improve on this here we use

$$
\left\|R_{n}\right\|_{n}=l u b_{\left\{\left\|w_{n}\right\|_{w}=1\right\}}\left[\sum_{k=0}^{n} E_{k}^{2}\right]^{1 / 2} .
$$


From Theorem 3.2 and (2.70) it will be shown that

$$
\left\|R_{n}\right\|_{n} \leq c n^{-r+1 / 2}
$$

so that

$$
\left\|u-v_{n}\right\|_{w} \leq c n^{-r+1 / 2} .
$$

Again, this appears to be the best that can be obtained using perturbation theory.

We now proceed to obtain the bounds on the integration errors needed to establish (2.32) and (2.72).

\section{Bounds on integration errors.}

Theorem 3.1. Let $w(x) \geq 0$ be a nonnegative integrable weight function, and let $\left\{\varphi_{k}\right\}$ be the orthogonal polynomials associated with $w$. Let $X_{n}=\operatorname{span}\left(\left\{\varphi_{k}\right\}_{k=0}^{n}\right)$ and $v_{n} \in X_{n}$ with $\left\|v_{n}\right\|=1$. Consider the integral

$$
I_{n}=\int_{-1}^{1} w(x) f(x) v_{n}(x) d x
$$

where $f \in C^{r}$. Suppose $I_{n}$ is approximated by $Q_{M}\left(f v_{n}\right)$ where $Q_{M}$ is an integration rule satisfying (2.53)-(2.54). Then the error

$$
e=I_{n}-Q_{M}\left(f v_{n}\right)
$$

satisfies

$$
|e| \leq c n^{-r}
$$

where $c$ depends only on $f$.

Proof. Since $\varphi_{k} v_{n} \in X_{2 n}, 0 \leq k \leq n$, and $Q_{M}(g)=I_{n}$ for all $g \in X_{2 n}, e\left(f v_{n}\right)=0$ for all $f \in X_{n}$. By Lemma 4.1 of [12]

$$
|e| \leq \inf _{y \in X_{n}}\|f-y\|_{\infty}\|e\|
$$


where $\|e\|$ is the norm of $e$ considered as a linear functional $C[-1,1]$.

By Jackson's theorem $[2]$

$$
\inf _{y \in X_{n}}\|f-y\|_{\infty} \leq c n^{-r}, \quad n>r .
$$

Thus we need to bound $\|e\|$. Hence

$$
|e| \leq \int_{-1}^{1} w(x)|f(x)|\left|v_{n}(x)\right| d x+\sum_{m=1}^{M} w_{m}\left|f\left(t_{m}\right)\right|\left|v_{n}\left(t_{m}\right)\right| .
$$

By the Cauchy-Schwarz inequality for integrals and sums

$$
\begin{aligned}
& \int_{-1}^{1} w(x) \mid f(x)|| v_{n}(x) \mid d x \\
& \leq {\left[\int_{-1}^{1} w(x) f^{2}(x) d x\right]^{1 / 2}\left[\int_{-1}^{1} w(x) v_{n}^{2}(x) d x\right]^{1 / 2} } \\
&= {\left[\int_{-1}^{1} w(x) f^{2}(x) d x\right]^{1 / 2} } \\
& \leq\|f\|_{\infty}\left[\int_{-1}^{1} w(x) d x\right]^{1 / 2}
\end{aligned}
$$

since $\int_{-1}^{1} w(x) v_{n}^{2}(x) d x=1$. Also

$$
\begin{aligned}
& \sum_{m=1}^{M} w_{m} \mid f\left(x_{m}\right)|| v_{n}\left(x_{m}\right) \mid \\
& \leq {\left[\sum_{m=1}^{M} w_{m}\left|f\left(x_{m}\right)\right|^{2}\right]^{1 / 2}\left[\sum_{m=1}^{M} w_{m} v_{n}^{2}\left(x_{m}\right)\right]^{1 / 2} } \\
& \leq\|f\|_{\infty}\left[\sum_{m=1}^{M} w_{m}\right]^{1 / 2} \\
& \quad=\|f\|_{\infty}\left[\int_{-1}^{1} w(x) d x\right]^{1 / 2}
\end{aligned}
$$

since $v_{n}^{2} \in X_{2 n}$ and $Q_{M}$ is exact for all $g \in X_{2 n}$. Thus

$$
|e| \leq 2\left[\int_{-1}^{1} w(x) d x\right]^{1 / 2}\|f\|_{\infty}
$$


giving

$$
\|e\| \leq 2\left[\int_{-1}^{1} w(x) d x\right]^{1 / 2}
$$

Hence $|e| \leq 2 c\left[\int_{-1}^{1} w(x) d x\right]^{1 / 2} n^{-r}, n>r . \quad \square$

Theorem 3.2. Let $\rho \geq 0, \gamma \geq 0$ be nonnegative weight functions on $[-1,1]$, and let $\left\{\varphi_{k}\right\}$ and $\left\{\chi_{k}\right\}$ be the orthogonal polynomials associated with $\rho$ and $\gamma$, respectively. Let $\kappa(x, t) \in C^{r}([-1,1] \times[-1,1])$ and consider the integrals

$$
I_{n}=\int_{-1}^{1} \int_{-1}^{1} \rho(x) \gamma(t) \kappa(x, t) z_{n}(x) v_{n}(t) d x d t
$$

where $v_{n} \in \operatorname{span}\left(\left\{\chi_{k}\right\}_{k=0}^{n}\right)$ and $z_{n} \in \operatorname{span}\left(\left\{\varphi_{k}\right\}_{k=0}^{n}\right)$ with $\left\|v_{n}\right\|_{\gamma}=$ $\left\|z_{n}\right\|-\rho=1$. Then the error

$$
E=I_{n}-Q_{M} \times Q_{N}\left(\kappa v_{n} z_{n}\right)
$$

satisfies

$$
|E| \leq c n^{-r}, \quad n>r
$$

where $c$ depends only on $k(x, t)$ and not on $n$.

Proof. By definition

$$
\begin{aligned}
E= & \int_{-1}^{1} \int_{-1}^{1} \rho(x) \gamma(t) \kappa(x, t) v_{n}(t) z_{n}(x) d x d t \\
& -\sum_{l=1}^{N} \sum_{m=1}^{M} \sigma_{l} w_{m} \kappa\left(x_{l}, t_{m}\right) v_{n}\left(t_{m}\right) z_{n}\left(x_{l}\right) .
\end{aligned}
$$

Letting

$$
h_{m}(x)=\kappa\left(x, t_{m}\right), \quad 1 \leq m \leq M,
$$


and

$$
\begin{gathered}
h(t)=\int_{-1}^{1} \rho(x) \kappa(x, t) z_{n}(x) d x \\
E=\int_{-1}^{1} \gamma(t) h(t) v_{n}(t) d t-\sum_{m=1}^{M} w_{m} v_{n}\left(t_{m}\right) Q_{N}\left(h_{m} z_{n}\right) .
\end{gathered}
$$

But

$$
\begin{aligned}
Q_{N}\left(h_{m} z_{n}\right) & =\int_{-1}^{1} \rho(x) \kappa\left(x, t_{m}\right) z_{n}(x) d x-e\left(h_{m} z_{n}\right) \\
& =h\left(t_{m}\right)-e\left(h_{m} z_{n}\right) .
\end{aligned}
$$

Using (3.19) in (3.18) gives

$$
\begin{aligned}
E= & \int_{-1}^{1} \gamma(t) h(t) v_{n}(t) d t \\
& -\sum_{m=1}^{M} w_{m} v_{n}\left(t_{m}\right)\left[h\left(t_{m}\right)-e\left(h_{m} z_{n}\right)\right] \\
= & \int_{-1}^{1} \gamma(t) h(t) v_{n}(t)+\sum_{m=1}^{M} w_{m} v_{n}\left(t_{m}\right) e\left(h_{m} z_{n}\right) \\
& -\sum_{m=1}^{M} w_{m} v_{n}\left(t_{m}\right) h\left(t_{m}\right) \\
= & e\left(h v_{n}\right)+\sum_{m=1}^{M} w_{m} v_{n}\left(t_{m}\right) e\left(h_{m} z_{n}\right) .
\end{aligned}
$$

By the proof of Theorem $3.1\left|e\left(h v_{n}\right)\right| \leq c_{1} n^{-r}$ and $\left|e\left(h_{m} z_{n}\right)\right| \leq c_{2} n^{-r}$ uniformly in $m$. (The uniformity in $m$ follows from the error formula in Jackson's theorem [3].) Thus

$$
|E| \leq c_{1} n^{-r}+c_{2} n^{-r} \sum_{m=1}^{M} w_{m}\left|v_{n}\left(t_{m}\right)\right| .
$$


By the argument in Theorem 3.1

$$
\sum_{m=1}^{M} w_{m}\left|v_{n}\left(t_{m}\right)\right| \leq\left[\int_{-1}^{1} \gamma(t) d t\right]^{1 / 2}
$$

so that

$$
|E| \leq c_{1} n^{-r}+c_{3} n^{-r} \leq c_{4} n^{-r}
$$

Summarizing our arguments in Section 2 and using Theorem 3.2 we get the following theorems.

Theorem 3.3. Let $v_{n}$ be the discrete Galerkin approximation to the solution of the Fredholm equation (2.1). If $f \in C^{r}$ and $\kappa \in C^{r}, r \geq 1$, then $v_{n}$ converges to $u$ in $L_{2}$ and $\left\|u-v_{n}\right\| \leq c n^{-r+1 / 2}$.

Proof. This follows from the error bound (2.25) and Theorem 3.2 with $\rho=\gamma=1, v_{n} \in X_{n}$ and $z_{n}=\varphi_{k}, 0 \leq k \leq n$.

Theorem 3.4. Let $v_{n}$ be the discrete Galerkin approximation to the CSIE (2.33). If $f \in C^{r}, \kappa \in C^{r}, r \geq 1$, then $v_{n}$ converges to $u$ in $L_{w}$ and $\left\|u-v_{n}\right\|_{w} \leq n^{-r+1 / 2}$.

Proof. This follows from the error bound (2.68) and Theorem 3.2 with $\gamma=w, \rho=1 / w, v_{n} \in X_{n}$ and $z_{n}=\chi_{k}, 0 \leq k \leq n$.

Remark. In [10] we analyzed a collocation method for the CSIE (2.33) with $u \cong u_{n}=\sum_{k=0}^{n} a_{k} \psi_{k}$ with $\left\{a_{k}\right\}$ obtained by setting $(H+K) u_{n}\left(x_{j}\right)-f\left(x_{j}\right)=0,0 \leq j \leq n$ where $\left\{x_{j}\right\}$ are the zeros of $\chi_{n+1}$. When the integrals $\left\{K u_{n}\left(x_{j}\right)\right\}$ were approximated by using $Q_{M}$ in (2.52), it was shown that the corresponding discrete collocation method $v_{n}$ converges to $u$ in $L_{w}$ and $\left\|u-v_{n}\right\|_{w} \leq c n^{-r+1 / 2}$. Theorem 3.4 shows that the discrete Galerkin and collocation methods appear to converge at the same rate. In [13] we showed that the discrete Galerkin method can be implemented using an 'almost sparse' matrix. This suggests that the discrete Galerkin method may be more efficient than 
the corresponding discrete collocation method. This is in contrast to the conventional wisdom on this subject $[\mathbf{1 1}]$.

One can define a similar discrete collocation method for (2.1) with the same $0\left(n^{-r+1 / 2}\right)$ convergence rate as for $v_{n}$.

5. A hypersingular equation. In recent years there has been considerable interest in hypersingular equations as a means for solving a variety of boundary value problems $[\mathbf{1 6}-\mathbf{1 8}]$.

For the one-dimensional equation

$$
\frac{1}{\pi} \frac{d}{d x} \int_{-1}^{1} \frac{\sqrt{1-t^{2}}}{t-x} u(t) d t+\int_{-1}^{1} \sqrt{1-t^{2}} \kappa(x, t) u(t) d t=f(x)
$$

a Galerkin method was developed by Frenkel in [6] and analyzed by Golberg in $[\mathbf{8}, \mathbf{9}]$. In those papers it was assumed that all the integrals were evaluated analytically. Here, using the results in Theorems 3.1 and 3.2 , we analyze the convergence of a discrete version of this algorithm where quadrature errors are taken into account. We begin by reviewing the analysis given in $[8]$.

For this we write (4.1) in operator form as

$$
H u+K u=f
$$

where

$$
H u=\frac{1}{\pi} \frac{d}{d x} \int_{-1}^{1} \frac{\sqrt{1-t^{2}}}{t-x} u(t) d t
$$

and

$$
K u=\int_{-1}^{1} \sqrt{1-t^{2}} \kappa(x, t) u(t) d t
$$

If

$$
U_{n}=\sin (n+1) \theta / \sin \theta, \quad \theta=\cos ^{-1} x,
$$

are the Chebyshev polynomials of the second kind, then [8]

$$
H U_{n}=-(n+1) U_{n}, \quad n \geq 0 .
$$


It then follows from (4.6) that $H$ can be extended as a bounded linear operator from $L_{1}(\rho) \rightarrow L(\rho)$ where $L(\rho)$ is the space of functions square integrable with respect to $\rho=\left(1-x^{2}\right)^{1 / 2}$ and $L_{1}(\rho)$ is the subspace of functions $u \in L(\rho)$ satisfying

$$
\|u\|_{1}^{2}=\sum_{k=0}^{\infty}(k+1)^{2}\left\langle u, \varphi_{k}\right\rangle_{\rho}^{2}<\infty
$$

where

$$
\varphi_{k}=\sqrt{\frac{2}{\pi}} U_{k}
$$

and

$$
\langle f, g\rangle_{\rho}=\int_{-1}^{1} \rho(t) f(t) g(t) d t
$$

If $k(x, t)$ is continuous, then $K$ defines a compact operator from $L_{1}(\rho) \rightarrow L(\rho)$ [8]. Thus, we consider solving (4.1) for $u \in L_{1}(\rho)$ for $f \in L(\rho)$. We assume that (4.1) has a unique solution for each $f \in L(\rho)$.

To solve (4.1) by Galerkin's method we approximate $u$ by

$$
u_{n}=\sum_{k=0}^{n} a_{k} \varphi_{k}
$$

where $\left\{a_{k}\right\}_{n=0}^{n}$ are obtained by solving

$$
\left\langle(H+K) u_{n}-f, \varphi_{j}\right\rangle_{\rho}=0, \quad 0 \leq j \leq n .
$$

Using (4.6) and (4.9) gives

$$
-(j+1) a_{j}+\sum_{k=0}^{n} a_{k}\left\langle K \varphi_{k}, \varphi_{j}\right\rangle_{\rho}=\left\langle f, \varphi_{j}\right\rangle_{\rho}, \quad 0 \leq j \leq n .
$$

In [9] it was shown that $u_{n} \rightarrow u$ in $L(\rho)$ and

$$
\left\|u-u_{n}\right\|_{\rho} \leq c n^{-r}
$$


if $f \in C^{r}$ and $k \in C^{r}$. It was also shown that if $r>3$ that $\| u-$ $u_{n} \|_{\infty} \leq c n^{-r+2}$. These results were obtained under the assumption that $\left\{\left\langle K \varphi_{k}, \varphi_{j}\right\rangle_{\rho}\right\}$ and $\left\{\left\langle f, \varphi_{j}\right\rangle_{\rho}\right\}$ were evaluated analytically. For practical implementation we consider quadrature rules $\left\{Q_{M}\right\}$ where

$$
\int_{-1}^{1} \rho(t) g(t) d t \simeq Q_{M}(g)=\sum_{m=1}^{M} w_{m} g\left(t_{m}\right)
$$

satisfying (2.53) and (2.54) and approximate

$$
\left\langle K \varphi_{k}, \varphi_{j}\right\rangle_{\rho} \simeq Q_{M} \times Q_{M}\left(\kappa \varphi_{k} \varphi_{j}\right)
$$

and

$$
\left\langle f, \varphi_{j}\right\rangle_{\rho} \simeq Q_{M}\left(f \varphi_{j}\right)
$$

Then using (4.14) and (4.15) in (4.11) $u_{n}$ is approximated by

$$
v_{n}=\sum_{k=0}^{n} b_{k} \varphi_{k}
$$

where $\left\{b_{k}\right\}_{k=0}^{n}$ satisfy

$$
\begin{aligned}
-(j & +1) b_{j}+\sum_{k=0}^{n} b_{k}\left[\sum_{m=1}^{M} \sum_{l=1}^{M} w_{l} w_{m} \kappa\left(x_{l}, t_{m}\right) \varphi_{k}\left(t_{m}\right) \varphi_{j}\left(x_{l}\right)\right] \\
& =\sum_{l=1}^{M} w_{l} f\left(x_{l}\right) \varphi_{j}\left(x_{l}\right),
\end{aligned}
$$

and $\left\{t_{m}\right\}$ and $\left\{t_{l}\right\}$ represent the same set of points.

Proceeding as in the Fredholm and singular cases, one can show that $v_{n}$ satisfies the operator equation

$$
H v_{n}+P_{n} K v_{n}+R_{n} v_{n}=P_{n} f+r_{n}
$$


where $P_{n}$ is the operator of orthogonal projection onto $\operatorname{span}\left(\left\{\varphi_{k}\right\}_{k=0}^{n}\right)$,

$$
R_{n} v_{n}=\left[\pi_{n} K_{n} v_{n}-P_{n} K v_{n}\right]=-\sum_{k=0}^{n} E_{k}\left(\kappa \varphi_{k} v_{n}\right) \varphi_{k}
$$

$$
\begin{aligned}
K_{n} v_{n} & =\sum_{m=1}^{M} w_{m} \kappa\left(x, t_{m}\right) v_{n}\left(t_{m}\right), \\
\pi_{n} u & =\sum_{k=0}^{n} Q_{M}\left(u \varphi_{k}\right) \varphi_{k}
\end{aligned}
$$

and

$$
r_{n}=\pi_{n} f-P_{n} f=-\sum_{k=0}^{n} e_{k}\left(f \varphi_{k}\right) \varphi_{k}
$$

where $E_{k}$ and $e_{k}$ are the quadrature errors in approximating $\left\langle K v_{n}, \varphi_{k}\right\rangle_{\rho}$ and $\left\langle f, \varphi_{k}\right\rangle_{\rho}$ by $Q_{M} \times Q_{M}\left(\kappa \varphi_{k} v_{n}\right)$ and $Q_{M}\left(f \varphi_{k}\right)$, respectively.

Using Theorem 1 of $[\mathbf{1 8}]$ to establish convergence of $v_{n}$, it suffices to show

$$
\left\|r_{n}\right\|_{\rho} \rightarrow 0
$$

and

$$
\left\|R_{n}\right\|_{n} \rightarrow 0
$$

where

$$
\left\|R_{n}\right\|_{n}=l u b_{\left\{\left\|w_{n}\right\|_{1}=1\right\}}\left\{\left\|R_{n} w_{n}\right\|_{\rho}\right\} .
$$

Since $\left\|w_{n}\right\|_{\rho} \leq\left\|w_{n}\right\|_{1}$

$$
\begin{aligned}
\left\|R_{n}\right\|_{n} & \leq l u b\left\{\left\|R_{n} w_{n}\right\|_{\rho}, w_{n} \in X_{n},\left\|w_{n}\right\|_{\rho} \leq 1\right\} \\
& =\operatorname{lub}\left\{\left\|R_{n} w_{n}\right\|_{\rho}, w_{n} \in X_{n},\left\|w_{n}\right\|_{\rho}=1\right\} \\
& =l u b_{\left\{\left\|w_{n}\right\|_{\rho}=1\right\}} \sum_{k=0}^{n}\left|E_{k}\left(\kappa \varphi_{k} w_{n}\right)\right|^{2} .
\end{aligned}
$$


Using Theorems 3.1 and 3.2 , we find that

$$
\left\|R_{n}\right\|_{n} \leq c_{1} n^{-r+1 / 2}
$$

and

$$
\left\|r_{n}\right\|_{\rho} \leq c_{2} n^{-r+1 / 2}
$$

if $\kappa \in C^{r}$ and $f \in C^{r}$. Using (4.27), (4.28) and (4.12) and the error estimate [18]

$$
\begin{aligned}
\left\|u-v_{n}\right\|_{\rho} & \leq\left\|u-v_{n}\right\|_{1} \\
& \leq c\left[\left\|u-u_{n}\right\|_{1}+\left\|R_{n}\right\|_{n}+\left\|r_{n}\right\|_{\rho}\right]
\end{aligned}
$$

we find that

$$
\left\|u-v_{n}\right\|_{\rho} \leq c n^{-r+1 / 2} .
$$

Thus, the discrete Galerkin method for (4.1) has a convergence rate analogous to that for Fredholm equations and CSIEs.

5. Fredholm equations on the sphere. The results given in Sections 2-4 are for one-dimensional equations. In this section we show how our previous analysis can be extended to analyze an algorithm developed by Atkinson for solving Fredholm equations on the sphere when the data are smooth.

For this, let

$$
D=\left\{(x, y, z) \in \mathbf{R}^{3} \mid x^{2}+y^{2}+z^{2}=1\right\}
$$

be the unit sphere in $\mathbf{R}^{3}$ and consider the integral equation

$$
u(\mathbf{P})=\int_{D} \kappa(\mathbf{P}, \mathbf{Q}) u(\mathbf{Q}) d s+f(\mathbf{P}), \quad \mathbf{P} \in D,
$$

where $f \in C^{r}(D)$ and $\kappa(\mathbf{P}, \mathbf{Q}) \in C^{r}(D \times D), r \geq 1$. We assume that (5.1) has a unique solution in $C^{r}$ for each $f \in C^{r}$.

In a series of papers Atkinson considered solving (5.1) using Galerkin's method with spherical harmonics as a basis [1-3]. Here we analyze the 
convergence of the discrete version of this algorithm using integration rules analogous to (2.9) for the one-dimensional case.

Now let $X_{n}=\operatorname{span}\left(\left\{Y_{l m}, 0 \leq l \leq n, 1 \leq m \leq 2 l+1\right\}\right)$ be the set of spherical polynomials of degree $\leq n$ where $\left\{Y_{l m}\right\}$ are the normalized spherical harmonics on $D[2]$. The dimension of $X_{n}$ is $N(n)=(n+1)^{2}$. As is well known, $\left\{Y_{l m}\right\}$ is an orthonormal basis for $X_{n}$.

Let $\left\{\hat{Y}_{1}, \cdots, \hat{Y}_{N}\right\}$ be an ordering of $\left\{Y_{l m}\right\}$ and approximate $u$ by

$$
u_{N}=\sum_{k=1}^{N} a_{k} \hat{Y}_{k}
$$

where $\left\{a_{k}\right\}_{k=1}^{N}$ are determined by solving

$$
a_{j}=\sum_{k=1}^{N}\left\langle K \hat{Y}_{k}, \hat{Y}_{j}\right\rangle a_{k}+\left\langle f, \hat{Y}_{j}\right\rangle, \quad 1 \leq j \leq N,
$$

and the inner product $\langle$,$\rangle is given by$

$$
\langle f, g\rangle=\int_{D} f(\mathbf{P}) g(\mathbf{P}) d s .
$$

In [2] it was shown that $u_{N} \rightarrow u$ in $L_{2}(D)$ and

$$
\left\|u-u_{N}\right\| \leq c n^{-r} \text {. }
$$

(In fact in [2] only $K: L_{2} \rightarrow C^{r}$ was needed, so that singular potential kernels satisfy the theory.)

As for the one-dimensional cases practical implementation of (5.2)(5.3) requires the numerical evaluation of the integrals in (5.3). To do this, suppose that $Q_{M}$ is a quadrature rule

$$
\int_{D} g(\mathbf{P}) d s \simeq Q_{M}(g)=\sum_{m=1}^{M} w_{m} g\left(\mathbf{P}_{m}\right), \quad \mathbf{P}_{m} \in D,
$$

satisfying

(1) $w_{m}>0, \quad 1 \leq m \leq M$

(2) $\quad Q_{M}(g)=\int_{D} g(\mathbf{P}) d s, \quad \forall g \in X_{2 n}$. 
One example of such a rule is

$$
Q_{M}(g)=\delta \sum_{i=1}^{2(n+1)} \sum_{j=1}^{n+1} w_{j} g\left(\theta_{j}, \phi_{j}\right)
$$

where $\delta=\pi /(n+1), \phi_{i}=i \delta$ and $\left\{w_{j}\right\}\left\{\cos \theta_{j}\right\}$ are the Gauss-Legendre weights and nodes on $[-1,1]$, where $g(\theta, \phi)=g(\sin \theta \cos \phi, \sin \theta \sin \phi, \cos \theta)$ is the representation of $g$ in spherical polar coordinates on $D$.

Then approximate

$$
\left\langle f, \hat{Y}_{j}\right\rangle \simeq Q_{M}\left(f \hat{Y}_{j}\right)
$$

and

$$
\left\langle K \hat{Y}_{k}, \hat{Y}_{j}\right\rangle \simeq Q_{M} \times Q_{M}\left(\kappa \hat{Y}_{k} \hat{Y}_{j}\right)
$$

This gives $v_{N} \simeq u_{N}$ where

$$
v_{N}=\sum_{k=1}^{N} b_{k} \hat{Y}_{k}
$$

and $\left\{b_{k}\right\}_{k=1}^{N}$ satisfy

$$
\begin{aligned}
b_{j}= & \sum_{k=1}^{N} b_{k}\left[\sum_{m=1}^{M} \sum_{l=1}^{M} w_{l} w_{m} \kappa\left(\mathbf{P}_{m}, \mathbf{Q}_{l}\right) \hat{Y}_{k}\left(\mathbf{Q}_{l}\right) \hat{Y}_{j}\left(\mathbf{P}_{m}\right)\right] \\
& +\sum_{l=1}^{M} w_{l} f\left(\mathbf{Q}_{l}\right) \hat{Y}_{j}\left(\mathbf{Q}_{l}\right),
\end{aligned}
$$

and $\left\{\mathbf{P}_{m}\right\}$ and $\left\{\mathbf{Q}_{l}\right\}$ represent the same set of points.

Letting $P$ be the orthogonal projection onto $X_{n}$,

$$
K_{N} u=\sum_{l=1}^{M} w_{l} \kappa\left(\mathbf{P}, \mathbf{Q}_{l}\right) u\left(\mathbf{Q}_{l}\right)
$$

and

$$
\pi_{N} u=\sum_{k=1}^{N} Q_{M}\left(u \hat{Y}_{k}\right) \hat{Y}_{k}
$$


$v_{N}$ satisfies

$$
v_{N}=P_{N} K v_{N}+R_{N} v_{N}+P_{N} f+r_{N}
$$

where

$$
R_{N} v_{N}=\left[P_{N} K-\pi_{N} K_{N}\right] v_{N}=\sum_{k=1}^{N} E_{k}\left(\kappa \hat{Y}_{k} v_{N}\right) \hat{Y}_{k}
$$

and

$$
r_{N}=-\left[P_{N} f-\pi_{N} f\right]=\sum_{k=1}^{N} e_{k}\left(f \hat{Y}_{k}\right) \hat{Y}_{k} .
$$

As in the one-dimensional cases, define

$$
\begin{aligned}
\left\|R_{N}\right\|_{N} & =l u b\left\{\left\|R_{N} w_{N}\right\|, w_{N} \in X_{n},\left\|w_{N}\right\|=1\right\} \\
& =l u b_{\left\{\left\|w_{N}\right\|=1\right\}}\left[\sum_{k=1}^{N} E_{k}^{2}\left(\kappa \hat{Y}_{k} w_{N}\right)\right]^{1 / 2},
\end{aligned}
$$

and

$$
\left\|r_{N}\right\|=\left[\sum_{k=1}^{N} e_{k}\left(f \hat{Y}_{k}\right)\right]^{1 / 2} .
$$

Then we can show that $v_{N} \rightarrow u$ provided that

$$
\left\|R_{N}\right\|_{N} \rightarrow 0 \text { and }\left\|r_{N}\right\| \rightarrow 0 .
$$

For this we need to bound the quadrature errors $E_{k}\left(\kappa \hat{Y}_{k} w_{N}\right)$ and $e_{k}\left(f \hat{Y}_{k}\right)$. This is done just as in Theorems 3.1 and 3.2 with a theorem of Ragozin replacing Jackson's theorem to bound the uniform approximation error of $f$ and $\kappa$ by spherical polynomials of degree $\leq n$. To avoid being repetitive, we will just state the relevant results and give a brief outline of the proofs.

Theorem 5.1. Let $f \in C^{r}(D), r>1$, and let $Q_{M}$ be a quadrature rule satisfying (5.7)-(5.8). Let $w_{N} \in X_{n}$ with $\left\|w_{N}\right\|=1$. Then the integration error

$$
e\left(f w_{N}\right)=\left\langle f, w_{N}\right\rangle-Q_{M}\left(f w_{N}\right)
$$


satisfies

$$
|e| \leq c n^{-r}, \quad r>1
$$

where $c$ depends only on $f$ and not on $n$.

Proof. Arguing as in Lemma 4.2 of [12] using (5.7)-(5.8) we find that

$$
|e| \leq c \inf _{g \in X_{n}}\|f-g\|
$$

where $c$ depends only on $f$ and not on $n$. By a theorem of Ragozin [19] there exists a function $h \in X_{n}$ such that

$$
\|f-h\|_{\infty} \leq c n^{-r}\|f\|_{r}
$$

where $\|f\|_{r}$ is the $C^{r}$ norm of $f$ given in [19]. Hence $\inf _{g \in X_{n}}\|f-g\|_{\infty} \leq$ $\|f-h\|_{\infty} \leq c n^{-r}\|f\|_{r}$ and using this in (5.22) gives (5.21).

Corollary 5.1. $\left\|r_{N}\right\| \leq c n^{-r+1}, r>1$.

Proof. Use (5.23) in (5.16).

Theorem 5.2. Let $\kappa(\mathbf{P}, \mathbf{Q}) \in C^{r}(D \times D), r>1$, and let $Q_{M}$ satisfy (5.7)-(5.8). If $w_{N} \in X_{n}$ and $z_{N} \in X_{n}$ with $\left\|w_{N}\right\|=\left\|z_{N}\right\|=1$, the integration error

$$
E\left(\kappa w_{N} z_{N}\right)=\left\langle K w_{N}, z_{N}\right\rangle-Q_{M} \times Q_{M}\left(\kappa w_{N} z_{N}\right)
$$

satisfies

$$
|E| \leq c n^{-r}, \quad r>1,
$$

where $c$ depends only on $\kappa$ and not on $n$.

Proof. Repeat the argument in Theorem 3.2 using Theorem 5.1 in place of Theorem 3.1 and using Ragozin's theorem in place of Jackson's theorem. 
Corollary 5.2. $\left|E_{k}\right| \leq c n^{-r}$ independent of $w_{N}$.

Proof. Letting $z_{N}=\hat{Y}_{k}, 1 \leq k \leq N$ in $E\left(\kappa w_{N} \hat{Y}_{k}\right)$, then (5.25) gives $|E| \leq c n^{-r}$ independent of $w_{N}$.

Corollary 5.3. $\left\|R_{N}\right\|_{N} \leq c n^{-r+1}$.

Proof. Using Corollary 5.2,

$$
l u b_{\left\{\left\|w_{N}\right\|=1\right\}}\left[\sum_{k=1}^{N} E_{k}^{2}\left(\kappa w_{N} \hat{Y}_{k}\right)\right]^{1 / 2} \leq c n n^{-r}=c n^{-r+1} .
$$

Using Theorems 5.1-5.2 one establishes the convergence of $v_{N}$.

Theorem 5.3. Let $f \in C^{r}(D) \kappa \in C^{r}(D \times D), r>1$, and $\left\{Q_{M}\right\}$ be a sequence of quadrature rules satisfying (5.7)-(5.8). Then $v_{N} \rightarrow u$ in $L^{2}(D)$ and

$$
\left\|u-v_{N}\right\| \leq c n^{-r+1} .
$$

Proof. From Corollaries (5.1)-(5.3) $\left\|r_{N}\right\| \rightarrow 0$ and $\left\|R_{N}\right\|_{N} \rightarrow 0$. Hence, it follows from

$$
\left\|u-v_{N}\right\| \leq c\left[\left\|u-u_{N}\right\|+\left\|R_{N}\right\|_{N}+\left\|r_{N}\right\|\right] \leq c n^{-r+1}
$$

and Corollaries (5.1)-(5.3) and (5.5) that $v_{N} \rightarrow u$ and $\left\|u-v_{N}\right\| \leq$ $c n^{-r+1}$.

Again, as in the one-dimensional cases, this appears to be the best bound that can be obtained by perturbation methods. For another approach to this problem, see [7].

Acknowledgment. The author would like to acknowledge the generous help of Professor C.S. Chen in the preparation of this paper. 


\section{REFERENCES}

1. K. Atkinson, The numerical solution of Laplace's equation in three dimensions, SIAM J. Numer. Anal. 19 (1982), 263-274.

2. - Algorithm 629: An integral equation program for Laplace's equation in three dimensions, ACM Trans. Math. Software 11 (1985), 85-96.

3. - A survey of boundary integral equation methods for the numerical solution of Laplace's equation in three dimensions, in Numerical solution of integral equations (M.A. Golberg, ed.), Plenum Publishing Co., New York, 1990.

4. K. Atkinson and A. Bogomolny, The discrete Galerkin method for integral equations, Math. Comp. 48 (1987), 595-616.

5. C.T.H. Baker, The numerical treatment of integral equations, Oxford University Press, Oxford, England, 1977.

6. A. Frenkel, A Chebyshev expansion of singular integrodifferential equations with a $\partial^{2}[\log |s-t|] / \partial s \partial t$ kernel, J. Comp. Phys. 51 (1983), 335-342.

7. M. Ganesh, I.G. Graham and J. Sivaloganathan, A pseudospectral $3 D$ boundary integral method applied to a nonlinear model problem from finite elasticity, SIAM J. Numer. Anal. 31 (1994), 1278-1414.

8. M.A. Golberg, The convergence of several algorithms for solving integral equations with finite-part integrals, J. Integral Equations 5 (1983), 329-340.

9. - The convergence of several algorithms for solving integral equations with finite-part integrals: II, J. Integral Equations 9 (1985), 267-275.

10. - Discrete projection methods for Cauchy singular integral equations with constant coefficients, Appl. Math. Comp. 33 (1989), 1-41.

11. - Introduction to the numerical solution of Cauchy singular integral equations in numerical solution of integral equations (M.A. Golberg, ed.), Plenum Publishing Co., New York, 1990.

12. - Discrete polynomially-based Galerkin methods for Fredholm integral equations, J. Integral Equations Appl. 6 (1994), 197-211.

13. - A Note on the sparse matrix representation of discrete integral operators, Appl. Math. Comp. 70 (1995), 97-118.

14. S. Joe, Discrete Galerkin methods for Fredholm integral equations of the second kind, IMA J. Numer. Anal. 7 (1987), 149-164.

15. P.A. Martin and F.J. Rizzo, On Boundary integral equations for crack problems, Proc. Roy. Soc. London, Ser. A 421 (1989), 341-355.

16. P.A. Martin, End-point behavior of solutions to hypersingular integral equations, Proc. Roy. Soc. London, Ser. A 432 (1989), 301-320.

17. - Exact solution of a simple hypersingular integral equation, J. Integral Equations Appl. 4 (1992), 197-204.

18. G. Miel, Perturbed projection methods for split equations of the first kind, Integral Equations Operator Theory 8 (1985), 268-275. 
19. D. Ragozin, Constructive polynomial approximation on spheres and projective spaces, Trans. Amer. Math. Soc. 162 (1971), 157-170.

20. A. Spence and K.S. Thomas, On superconvergence properties of Galerkin's method for compact operator equations, IMA J. Numer. Anal. 3 (1983), 253-271.

2025 University Circle, Las Vegas, NV 89119-6051 